\title{
SARS CoV-2 Vaccination Autoimmunity, Antibody Dependent Covid-19 Enhancement 知䘠 and Other Potential Risks: Beneath the Tip of the Iceberg
}

Mina T Kelleni*

Pharmacology Department, Minia University, Egypt

Submission: August 04, 2021; Published: August 27, 2021

*Corresponding author: Mina T Kelleni, MD, PhD, Pharmacology Department, College of Medicine, Minia University, Egypt

\begin{abstract}
Nucleic acid based-mRNA based and adenovirus vectored-vaccines, were first ever or first commercially ever approved for the public, respectively. However, these newly emergency approved types possess a potential risk to induce auto-immune diseases e.g., thrombocytopenia, myocarditis and immune induced thrombosis and thromboembolism that might be fatal and could reason for some of the post vaccination sudden death reports. Moreover, all SARS CoV-2 types of vaccines, depending on the spike protein immunogenicity, especially the conventional inactivated ones might increase the likelihood of COVID-19 severity upon re-infection through antibody dependent enhancement which might reason for the recently described abundance of hospital admissions within seven days of vaccination and might also reason for some of the serious adverse effects encountered with administration of convalescent plasma to COVID-19 patients as well as they might share in development of some lethal SARS CoV-2 variants. Importantly, we suggest that SARS CoV-2 mass vaccination campaigns were the worst ever decision made and that making these COVID-19 vaccines compulsory or administering them to children or pregnant participants might be considered as a crime against humanity to the extent that no prior companies- governmental agreements would ever secure impunity. Finally, a full informed personalized risk benefit ratio especially for some described high-risk groups must be secured while suggesting that the subunit vaccines are the least hazardous ones.
\end{abstract}

Keywords: COVID-19; SARS CoV-2 vaccines; Autoimmune diseases; Antibody dependent enhancement

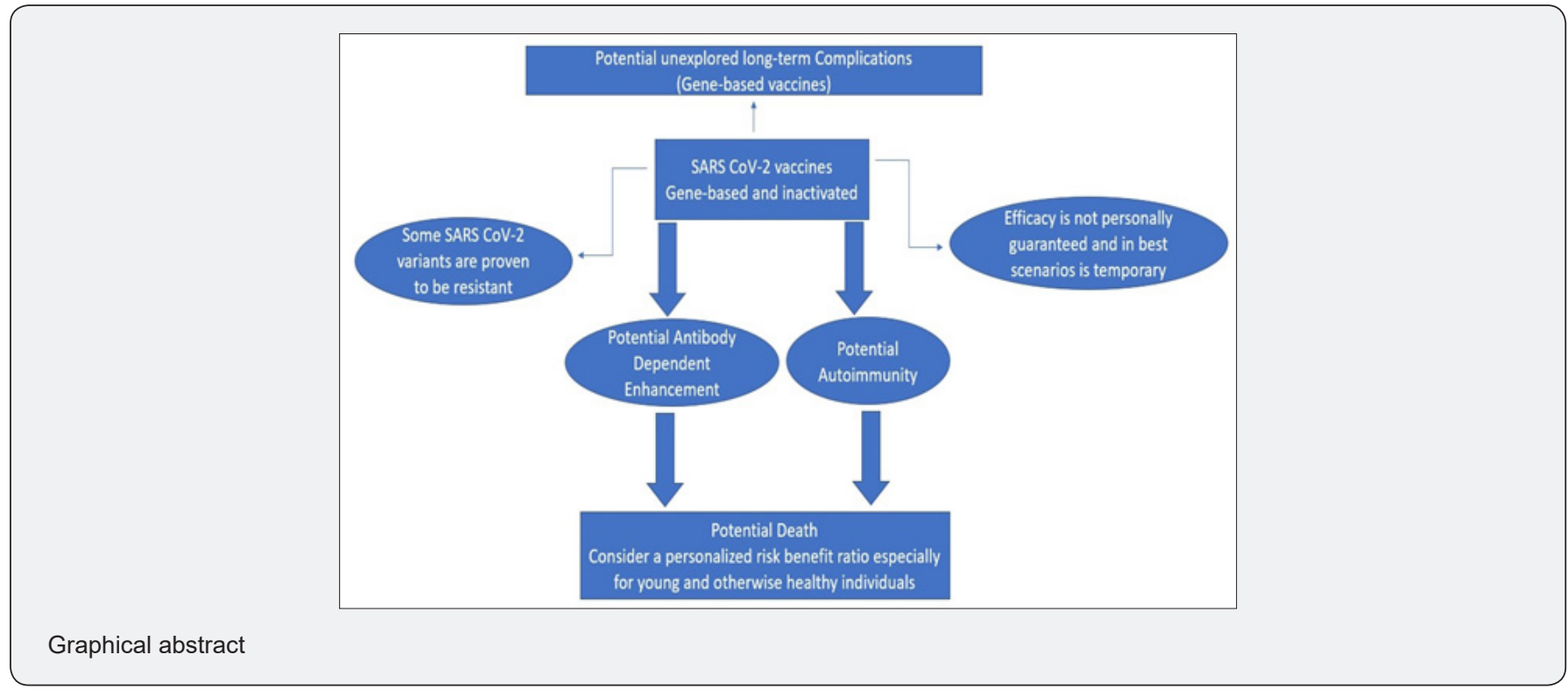




\section{Highlights}

I. Since August 2020 and until August 04, 2021, numerous "reputable" medical journals have denied this manuscript a fair opportunity to be peer reviewed.

II. Some SARS CoV-2 vaccines have been linked to acute serious/fatal adverse effects and all have not been fully approved, yet.

III. SARS CoV-2 induced antibody dependent enhancement has not been excluded yet.

IV. An informed personalized risk benefit ratio before receiving SARS CoV-2 subunit vaccines must be secured before administration to high-risk groups.

V. SARS CoV-2 mass vaccination campaigns with nucleic acid-based vaccines are the worst ever decision made, and long-term complications should never be undermined.

\section{Introduction}

Safe COVID-19 vaccines are considered of utmost importance to stem SARS CoV-2 current pandemic [1]. However, the unprecedent accelerated timelines to develop COVID-19 vaccines have necessitated a critical call for active pre- and post-licensure safety surveillance systems to properly investigate potential adverse effects or toxicities [1-3]. Importantly, whether the incidence of SARS CoV-2 vaccine related serious adverse effects might be considered rare or less rare $[4,5]$, or very difficult to be prove causation [6], the scientific community has an obligation to continue developing new standards for safety monitoring.

Notably, in a formal British report entitled: "Hospitalized vaccinated patients during the second wave, update April '21", even the high risk groups to develop COVID-19 complications might not eventually benefit from SARS CoV-2 vaccines as previously was expected and repeatedly advertised and we consider this report though considered of low evidence, at least currently, a rare one as regards to its high scientific integrity which is free of a potential economic bias. Similarly, almost half of the deaths in UK due to the delta variant were among fully vaccinated patients above 50 years old as reported in June 2021. Moreover, the Oxford/AstraZeneca ChAdOx1 nCoV-19 vaccine has been recently shown to be ineffective as regards to prevention of mild-moderate COVID-19 due to the B.1.351 South African variant [7].

Similarly, though all the propaganda that promotes studies with serious limitations and bias, scientific integrity still emphasizes the known fact that the current COVID-19 systemic vaccination is not likely to prevent nasal SARS-CoV-2 infection and asymptomatic transmission [8]. In this manuscript, we briefly discuss the potential autoimmune adverse effects of SARS CoV2 nucleic acid-based vaccines; adenovirus vectored and mRNA vaccines. Furthermore, we also briefly discuss the potential risk to develop more severe COVID-19 upon SARS CoV-2 reinfection after vaccination as compared to the natural infection; a phenomenon called antibody dependent disease enhancement and its potential association with adverse effects encountered while convalescent plasma was administered to COVID-19 patients.

Finally, we illustrate, from our point of view, some of the higher risk groups to develop autoimmune disorders urging that they might consider a personalized risk benefit ratio as well as some potential tools that might decrease this potential. We wish to confirm that the public has a moral, legal, and constitutional right to know all the potential hazards of COVID-19 newly emergency approved vaccines including even the rarest ones to allow an informed personalized risk benefit ratio to be weighed to freely decide whether to receive any or not. This right should never be argued or suppressed as, unfortunately, it appeared as the case when a professional peer review for this and other related preprinted manuscripts has been denied a non-biased peer review opportunity by numerous well reputed medical journals since August 2020 (Nature Medicine was the first in a long row "Why smokers should not hurry to be vaccinated with SARS CoV-2 mRNA vaccine?" (NMED-C108161) when I first tried to submit it to that journal before its first preprint at Authorea [9].

Similarly, and unfortunately, when a paper of other authors entitled: "The Safety of COVID-19 Vaccinations-We Should Rethink the Policy" miraculously escapes an apartheid wall and published while stating that "we have to accept four fatal and 16 serious side effects per 100,000 vaccinations in order to save the lives of 2-11 individuals per 100,000 vaccinations. For three deaths prevented by vaccination we have to accept two inflicted by vaccination" and the concludes that: "This lack of clear benefit should cause governments to rethink their vaccination policy", it was not unexpected that a retraction would be a biased inevitable outcome, but the surprise was that their reasons are so subtle, non-essential and the journal has even shamefully denied the authors their right to publish their reply.

\section{Adenovirus Vectored Vaccines Potential Autoimmu- nity Risk}

Autoimmunity developing due to similarities between viral and human proteins is one of the known sequalae of viral infections that include short term and sometimes permanent damage to the CNS [10]. Moreover, an increased autoimmunity risk was hypothesized due to the inclusion of new adjuvants into the already approved licensed vaccines [11]. However, this risk associated with COVID-19 vaccines especially the newly approved SARS CoV-2 ones is yet to be discovered. Notably, adenovirus vectored SARS CoV-2 vaccine has been first commercially approved to be used in humans in Russia which is currently undergoing a mass vaccination program and on December 30,2020, it has also been announced to be authorized for emergency supply in the UK 
followed by other countries and since that date millions of jabs have been administered basing on emergency not full approval.

Importantly, two adenovirus vectored SARS CoV-2 vaccine global phase III clinical trials were temporarily paused due to reports of serious adverse medical events of autoimmune and/ or inflammatory complications including multiple sclerosis and transverse myelitis which were ultimately deemed to be unrelated to the SARS CoV-2 vaccine. Moreover, lack of transparency concerns have been raised as the involved companies declined the release of the thorough details of these serious adverse events claiming patients' privacy issues [12-15] and a sharp criticism of the analysis of the results of one trial including a serious dose mistake that involved thousands of patients, claimed later to be a "beneficial" one, has also been raised [16].

Importantly, supraphysiological expression levels of spike proteins in some individuals who receive nucleic acid based vaccination might share in development of autoimmune reactions [17] and we recommend that the dose of the nucleic acid based vaccines, if decided to be received, should be optimized to the lowest possible dose and potential tools to prevent induced autoimmunity should be further developed and tested. In addition, we also suggest that a skewed immune virus spike proteinantibody complex might trigger and reason, at least partly, for this potential autoimmunity [18].

\section{mRNA Vaccines Potential Autoimmunity Risk}

mRNA based vaccines, first approved in UK for COVID-19 as a first ever approval for this novel type of vaccination in a western country to be followed by USA, the European Medicines Agency (EMA) as well as several countries worldwide, possess multiple theoretical and manufacturing advantages over traditional subunit, live attenuated and killed virus vaccines [19-21]. However, their remarkable high efficacy in SARS CoV-2 clinical trials contradicted the results of other previous clinical trials using mRNA vaccines to prevent H10N8, H7N9 influenza and rabies viruses which have been lower than what was expected when compared to those of their preclinical studies [20].

Moreover, though mRNA vaccines encoding HIV and CMV antigens elicited antigen specific CD4+ and CD8+ T cell immune responses; no reduction in viral load was observed [19]. Importantly, potential risks of mRNA, and saRNA, based vaccines include risk of autoimmunity due to development of autoreactive antibodies of any non-native nucleotides and delivery system components. Furthermore, the identification of individuals at an increased risk of autoimmune reactions before mRNA vaccination was advised [20,22,23]. Notably, other than the currently known potential risks of anaphylaxis or Bell's palsy present at the CDC website, soon after mRNA-based SARS CoV-2 vaccine approval, the Norwegian Medicines Agency started to investigate the potential causation of Pfizer-BioNTech mRNA (BNT162b2) vaccine against Covid-19 and the death of 75-year-old and elder 33 recipients.

Similarly, the Paul Ehrlich Institute in Germany has been reported to investigate 10 fatalities that occurred within four days of vaccination and whose age groups were not revealed to the public but described as previously seriously ill patients suffering from many underlying diseases [24]. Alarmingly, though attributing these fatalities to commonly encountered adverse effects in the elderly is usually advocated, yet an American 12-year-old female volunteer for BNT162b2 has suffered paralysis (transverse myelitis? Guillain-Barré?) and a piece of news of her was only publicly released very late and only after hundreds of millions of jabs have been administered. Similarly, an otherwise healthy 56-year-old American obstetrician and gynecologist has developed autoimmune thrombocytopenia three days after receiving BNT162b2 vaccine and later he was deceased of brain hemorrhage as a complication to this autoimmune disease.

Similarly, another American 60-year-old X-ray technologist was deceased four days after taking his second dose of the BNT162b2 vaccine, he complained of an acute abdominal pain and dyspnea and tested negative for COVID-19, later his condition deteriorated, was put into a medically induced coma and a ventilator. He eventually suffered from severe hypotension before death. Other than overweight and hypertension, he has not complained of any concomitant disorder. Moreover, at least one participant in the clinical trials has suffered from cardiac arrest and an otherwise healthy 41-year-old Portuguese nurse was found dead two days after receiving BNT162b2 vaccine. Similarly, an analysis published at Nakim.org has seriously doubted the integrity of the safety data reported by the Israeli ministry of health as regards to its adopted policy for mass vaccination with BNT162b2 vaccine and only an informal weak criticism of this report has confirmed the validity of its statistics.

Additionally, another report claims that post BNT162b2 mass vaccination increased Israeli all-cause mortality with an observational "murky wave of heart attacks" as well as suggestions of intended official lack of transparency. Interestingly, thought the official statements denies a single post SARS CoV-2 vaccination mortality, several unofficial Israeli reports strongly contradict this claim. However, Israeli authorities have announced a probable link between the second dose of BNT162b2 vaccine and myocarditis in young men aged 16 to 19 than in other age groups and I recommend independent international investigations for the best interests of transparency and this should also apply to the alarming claims about the actual potential SARS CoV- 2 vaccine related mortalities or that the CDC has manipulated the actual number or why the FDA has chosen not to require SARS CoV-2 vaccines' manufacturers post marketing more rigorous safety data capturing as claimed by a pioneer of the mRNA vaccine technology Dr. Robert Malone. 
Adenovirus Vectored and mRNA Vaccines Mutual

\section{Autoimmune Risks}

Importantly, immune thrombocytopenia was previously attributed to IgG opsonized dengue virus complexes bound to Fc receptors in platelets which were also suggested to play a central role in development of antibody dependent enhancement during dengue infection [25] and we suggest that the same mechanism might also apply to reason for the reported post BNT162b2 and Moderna mRNA-1273 SARS CoV-2 mRNA vaccination induced thrombocytopenia that also led the EMA to start a review of safety signal in patients who received any of BNT162b2, mRNA1273 and Oxford/AstraZeneca (ChAdOx1 nCOV-19) (adenovirus vectored) vaccines.

Moreover, autoantibody induced thrombosis was previously described in another setting [26] as well as other potential mechanisms for immunothrombosis [27] and venous thromboembolism was shown to be consistently associated with autoimmune diseases [28]. Taken together, we suggest that a dysregulated autoimmunity might be triggered in some, genetics might play a role, individuals who received SARS CoV-2 vaccines leading to sudden death from thromboembolism. Notably, though ChAdOx1 nCOV-19 vaccine has been first put under investigations because of multiple simultaneous fatality reports that led some European countries to halt its administration, permanently, or temporarily, as becoming usual, while claiming unscientifically valid similar incidence in the general population as the abstract facts refute this claim and declare that these vaccine related extremely serious adverse effects are more frequent than would be expected by chance.

Notably, arterial, venous thrombotic, or embolic events were recently reported in the South African Ad26.COV2.S Vaccine Study [5] and the reported cerebral venous sinus thrombosis, encountered post ChAdOx1 nCOV-19 vaccination was previously described with autoimmune thyroiditis/hypothyroidism [29] and its risk factors include the presence of autoantibodies like antiphospholipid and anticardiolipin antibodies [30]. Moreover, some scientists from Norway and Germany have independently confirmed the ability of ChAdOx1 nCOV-19 to trigger this autoimmune reaction and later AstraZeneca was instructed to flag a possible thrombotic side-effect of ChAdOx1 nCOV-19 vaccine on labelling and recently, vaccine-induced immune thrombotic thrombocytopenia was coined to describe the pathogenesis of some of these cases [31].

Ironically, the same sequence of denial, investigations occurred with the Johnson \& Johnson adenovirus vectored SARS CoV-2 Ad26.COV2-S vaccine as the FDA initially declared no causal thrombosis relationship is found, but fortunately a prompt vigilant decision of a temporary pause of Ad26.COV2-S vaccine until further evaluation was issued. Notably, we have formally contacted the FDA before this pause emailing a draft of this manuscript and later, we urged it to respond like EMA and wisely they did. Interestingly, after some European countries have fully suspended the use of ChAdOx1 nCOV-19 vaccine, UK has restricted its administration to people under 40 years old (previously under 30 years old) and started an analysis of its association with the autoimmune Guillain-Barre syndrome. Recently, the FDA has wisely labelled the Johnson \& Johnson Ad26.COV2-S vaccine with a potential increased risk of Guillain-Barre syndrome.

Unsurprisingly, British scientists have recently exposed in a preprint post SARS CoV-2 vaccines thrombotic events are not limited to the cerebral vasculature as splanchnic and portal vein thrombosis, with similar case fatality rate $(18.8 \%$ versus $20 \%$ of cerebral venous thrombosis), within two weeks post vaccination are more common with BNT162b2 and mRNA-1273 vaccines (44.9 per million versus 1.6 per million for their ChAdOx1 nCOV19 vaccine) and though they have mentioned that the incidence is much higher after COVID-19 but we suggest that their comparison is not out of bias especially when properly adjusted for the affected age and gender [32,33] and one may wonder what else might be discovered by other researchers.

Moreover, we would like to suggest that a fatal autoimmune myocarditis, which is known to be underdiagnosed, might also be responsible for some of the post SARS CoV-2 mRNA vaccination sudden death reports which are being attributed to other conditions to acquit mRNA vaccine while they might be due to vaccine related myocarditis causing fatal arrhythmias, acute-onset heart failure with cardiogenic shock or pericardial effusion with cardiac tamponade [34,35]. Notably, a 19-year-old Israeli patient suffered from tachycardia, dyspnea, and angina like pain after receiving his second dose of BNT162b2 vaccine to be hospitalized five days later with a confirmed diagnosis of myocarditis.

Importantly, since IL- 6 has been suggested to play an integral role in the pathogenesis of clinical and experimental viral myocarditis [36,37], we would like to suggest that the potential clinical benefits of few days administration of NSAIDs [38] with SARS CoV-2 vaccines either concomitantly or on the day after both the first and second (if there is one) jabs might eventually exceed the inconclusive potential risk to lower the immune response developed from the vaccines [39]. Recently and fortunately, EMA has begun an investigation to assess the association between SARS CoV-2 mRNA vaccines and myocarditis though starting with the usual declaration that no indication at present that these cases were due to the vaccines and most recently whistleblowers have revealed that FDA discuss in closed rooms a Canadian report showing Moderna vaccine linked to myocarditis more than that of Pfizer's and even a Pfizer PI has admitted in a paper entitled: "Myocarditis After SARS-CoV-2 Vaccination: True, True, and... Related?" that if these claims were confirmed, this would be the first of its kind adverse effect coming from a "non-live" vaccines. 
Moreover, an immunopathological phenomenon called antibody dependent enhancement (ADE) that might increase COVID-19 severity, discussed later, should be tested for a potential concomitant correlation in susceptible individuals e.g. some vaccines recipients who were previously primed by either SARS CoV-2 as silent infection or possibly through other commonly encountered corona viruses, might express an autoimmune lung reaction which was suggested to reason for COVID-19 pathogenesis $[10,40]$ and we suggest it might better suit COVID-19 complications whether or not linked to vaccination. Furthermore, we would like to recommend CDC to urgently change its neutral recommendation and to advice against administration of nucleic acid-based vaccines to persons complaining from autoimmune diseases.

\section{BNT162b2 Vaccine Potential Extra Risk}

Importantly and unfortunately, the sequence used in BNT162b2 vaccine was suggested to induce misleading errors in translational decoding and protein synthesis which were hypothesized to produce serious long-term health damage including neurodegenerative diseases and multiple sclerosis [41]. Furthermore, several adjuvants, newly used in vaccines, are known to trigger the innate and adaptive immune system with a theoretical, not confirmed, risk to induce autoimmune diseases [11] and since most of the discussed serious adverse effects and fatalities were reported with the BNT162b2 vaccine, there is a likelihood for at least a short-term potential extra hazard that might be a company specific, to be fully explored and compared as regards to its sequence and used adjuvants to its mRNA-1273 counterpart as an essential component of any investigation.

We also recommend consideration a sustained monitoring of the emergency approval granted by the FDA to BNT162b2 vaccine until all the claims against its design and its potential causation of some of the reported deaths are investigated and discussed.

\section{Antibody Dependent COVID-19 Enhancement} Potential Risk: Vaccines and Convalescent Plasma Links

Importantly, a risk for all types of SARS CoV-2 vaccines, especially the inactivated ones, that aim to develop antibodies against its spike protein is an immunopathological well recognized phenomenon called ADE which was reported and described with other respiratory and corona viruses including SARS CoV and MERS $[10,42]$. It was previously reported that in the presence of vaccine-elicited antiviral antibodies, SARS-CoV displayed an altered tropism toward primary human immune cells which were otherwise refractory to the virus. Furthermore, vaccines developed against animal coronaviruses has demonstrated an immune enhancement of disease in vaccinated recipients [43].

Importantly, individuals suffering from severe COVID-19 were suggested to be primed by one or more prior coronavirus exposures, and due to antigenic epitope heterogeneity, are experiencing the effects of ADE similar to that previously postulated with SARS CoV [44]. Additionally, recurrent COVID-19 infection was described, in a significant minority due to a variable immune response, to be more severe and potentially fatal [45] and SARS CoV-2 vaccines were also suggested to possess the same immunological risk and a modification of their design was suggested to lower the potential risk [10] to be noted that abnormal immunological response to SARS CoV-2 BNT162b2 vaccine has been described and most likely predisposed to an accelerated SARS CoV-2 induced mortality [46].

Notably, a higher antibody titre against SARS-CoV-2 being was associated with more severe disease and suggested to be linked to ADE as one possible probability that was not excluded by the other suggested mechanisms. Moreover, several studies in murine and non-human primate models for SARS-CoV vaccines showed enhanced immunopathology, enhanced respiratory disease [47] or skewing immunological or inflammation-resolving response $[42,48,49]$ on challenge with SARS CoV after immunization and thus the benefit of using SARS-CoV vaccine in humans was doubted [50] and a very interesting commentary that unfortunately has been unnoticed, possibly because of multiple prior rejections at more visible journals, has tested the outcomes of SARS CoV2 infection in 33 African green monkeys which were vaccinated with mRNA SARS CoV-2 vaccines and ARDS has developed in one [51].

Moreover, it was recently announced that $60 \%$ of seriously ill COVID-19 Israeli patients were vaccinated with BNT162b2 vaccine and we suggest this might be considered as potential $\mathrm{ADE}$ as well as a clear evidence of the inefficacy of the current COVID-19 vaccines at least against the delta variant. Similarly, $70 \%$ of new COVID-19 Israeli cases were reported, at least once, to be in the vaccinated people and it was suggested that natural recovery from past COVID-19 is more protective against infection from the Delta Variant than vaccination.

Accordingly, we disagree with Fu et al. [52] in their suggestion that an early, sub-optimal neutralizing antibody activity reasons for ADE responsible for the severe SARS CoV induced pulmonary disease and with Lee et al. [47] in their suggestion, basing on interpretations of some murine models findings, that SARS CoV2 vaccines that elicit high neutralizing antibody titres have a minimal risk of ADE which is supported by a preprinted study that used a SARS CoV-2 murine model [53] as neutralizing antibodies are described to induce ADE $[48,54]$ and a SARS CoV-2 DNA study that has been performed in non-human primates and frequently cited to acquit COVID-19 from ADE potential has clearly stated that it was not designed to examine safety issues.

Furthermore, it recommended future studies to specifically address the probability of enhanced respiratory disease due to ADE implying that their favorable impression should never be cited as potentially conclusive [55]. Importantly, another argument that 
is also used as a principle to refute or underestimate COVID-19 $\mathrm{ADE}$ risk is that antibodies can have very different properties in animals compared to those in the human host, because of altered functional species-specific interactions between the antibody and immune cells [56].

However, this should be used likewise in favor of the contradictory perspective, and we also might likewise suggest that results coming from non-corona viruses should not be considered of much significant value when trying to interpret the potential risk of ADE in COVID-19. Moreover, we suggest that while COVID-19 does not worsen after treatment with plasma from convalescent patients [56], it should not considered conclusive in a context that underestimate COVID-19 potential ADE risk for two reasons; the first is the timing as these antibodies are being administered to combat an undergoing infection and the other is that these antibodies might have worsened COVID-19 if proper validation of the reported cardiac events was conducted and thus an early ADE should not be excluded [57].

Interestingly, abundance of hospital admissions was described within seven days post SARS CoV-2 vaccination was described in the rare formal previously mentioned British document, and it was hypothesized that this might occur with recently asymptomatic SARS CoV-2 infected patients who received the vaccines and we recommend investigating this hypothesis as it might eventually appear as a mild form of ADE. Alarmingly, unlike the one year spent to develop a vaccine for SARS CoV-2 (a single-stranded RNA virus), the journey to develop RSV (another enveloped non-segmented single stranded RNA virus) vaccine took more than 60 years and has not ended yet. More alarmingly, $80 \%$ of young infants previously vaccinated with inactivated RSV who have been subsequently infected with wild RSV experienced enhanced respiratory disease that required hospitalization and two died and [58] and an atypical measles illness accompanied by peripheral edema and pneumonia occurred in ten children who had received inactivated measles (a third enveloped nonsegmented single stranded RNA virus) vaccine five to six years earlier, and significant pleural effusions were noted in three of them [59].

Importantly, we wish to strongly recommend against any suggested administration of any SARS CoV-2 vaccine to children, especially the inactivated ones. However, we confirm our recommendation using the lowest possible vaccination dose optimized to produce high-affinity anti-SARS CoV-2 IgG as this might be our route to decrease this and other potential likelihoods. Additionally, we reconfirm the need for developing suggested neutralizing nanobodies as well as new immunofocusing vaccines basing on the spike, $\mathrm{N}$ or other potential SARS CoV-2 immunological targets [42].

Finally, though reports of SARS CoV-2 infection early after vaccination have not reported $\mathrm{ADE}[60]$, yet the recent terrible surge of COVID-19 mortality in India should be further investigated whether SARS CoV-2 B.1.617 variants are the sole culprit or ADE might also be involved and whether the enthusiastic Indian vaccination program might promote the emergence of more lethal variants [61] and we anticipated on May 14, 2021 that the Indian Hades might be repeated in other countries unless prompt measures are executed, of course in vain [62]. Notably, the timing of re-infection or vaccination might play a factor in development of $\mathrm{ADE}$ as well as some individualized immune-genetic factors and thus, from our point of view, a call for close and vigilant follow up should not be ignored and any report of such adverse effect should not be underestimated.

\section{The Potentially Higher-Risk Groups and Potential Amelioration of The Risks}

Notably, we would like to explore some groups of individuals who are potentially more vulnerable to autoimmune diseases, aiming to recommend a personalized risk benefit ratio to be considered before a decision to be immunized by adenovirus and RNA based SARS CoV-2 vaccine until encouraging post marketing safety data are revealed for all SARS CoV-2 types of vaccines. The first higher-risk group are female [63] and this is a non-modifiable risk factor. However, reports of post SARS CoV-2 vaccination myocarditis seem to show male predominance in adolescents and young adults age 16 years or older and the initial female predominance in reports of vaccine-induced immune thrombotic thrombocytopenia might have been skewed by the demographics of early vaccinated populations.

The second group are smokers as cigarette smoke has been reported to lead to an enhanced risk of inflammatory and autoimmune diseases [64]. Notably, smokers are more likely to develop critical COVID-19 requiring mechanical ventilation [65] that might lead to a higher mortality rate $[66,67]$. Interestingly, alarms about the danger of misreading non-significant or inconclusive frequentist results containing several possible biases of a contradictory hypotheses have been raised $[68,69]$. Two other important groups that might be closely monitored include obese and diabetic individuals; obesity was suggested to be a major environmental factor contributing to the onset and progression of autoimmune diseases [70] and a concomitant autoimmune disease was encountered as 1 in 4 of 179,248 people diagnosed with type 1 diabetes [71]. Notably, a meta-analysis has showed diabetes, but not obesity, to be linked to a higher COVID-19 mortality [72].

However, increasing risks of COVID-19 hospital death were noticed to be associated with increasing levels of obesity (BMI $>40$ fully adjusted HR 2.27, 95\% CI 1.99-2.58) [73] and an informed personalized risk benefit ratio must be secured. Interestingly, quitting smoking at diagnosis was recently shown to decrease the risk of death in cancer patients [74], and quitting smoking was suggested to alleviate its impact in patients with pneumonia and 
other COVID-19 associated infections $[67,69,75]$, thus a beneficial advice to quit smoking together with another to lose overweight and to control the blood glucose levels might also help to lower the chances of SARS CoV-2 adenovirus and RNA-based vaccine potential autoimmunity in those individuals.

Mostimportantly, wewould liketo stress the utmostimportance to urge the participants to report all experienced adverse effects to a well-prepared post marketing surveillance system. Further, the search to improve methods that help to develop nucleic acidbased vaccines with minimal autoimmune potential risk should continue. However, as evolving post marketing safety concerns are released, we recommend considering an individualized risk benefit ratio especially for those higher risk groups of patients and we suggest that the subunit vaccines e.g., Novavax NVXCoV2373 vaccine are probably the least hazardous ones and it was a real global misfortune to fuel mRNA vaccines with 500 million dollars of tax payers money because of their mutual link with Dr. Anthony Fauci, if a decision to go for subunit vaccines, thousands of innocent lives could have been saved as we claim.

\section{Conclusion}

In conclusion, we totally condemn, from a medical point of view, any national policy that necessitates these experimental vaccines and we also condemn the European Court of Human Rights shameful ruling that compulsory vaccination would not contravene human rights law. Moreover, though "experts" finally admit that their claimed vaccine induced herd immunity is very unlikely [76] or almost impossible, they continue to make it almost compulsory and even advocate for vaccine passports. Alarmingly, we condemn the trials made by some pharmaceutical companies to test those vaccines in children as well as their attempt to seek clearance of usage in children aged two years and above as other than the discovered potential short-term complications, long term ones are not excluded as well [41].

Unsurprisingly, serious violations and manipulations of the trial protocol by which Pfizer has obtained FDA emergency authorization for administering its BNT162b2 vaccine to children have been published at americasfrontlinedoctors.org with no official reply and recently other researchers have called for a reconsideration of the current "political" trend to vaccinate the children with SARS CoV-2 vaccines as they suggested a huge outbalance in their risk benefit ratio [4].

Alarmingly, NEJM has rejected to publish a logical comment that heavily criticized the integrity of the post SARS CoV-2 vaccination spontaneous abortion of $12.6 \%$ that came through counting 700 participants who received their first jab in the third trimester i.e. at a time where no spontaneous abortion can occur [77] and unfortunately the authors had no alternative but to publish their comments revealing an actual rate of $82 \%$ at a nonacademic website and it was even later deleted. Notably, I was so fortunate to expose a similar NEJM bias, though for potentially toxic drugs, at an honorable journal [78]. Moreover, we would like to recommend following up the babies born to pregnant participants as long-term complications cannot be excluded and we consider their mothers' vaccination with these SARS CoV-2 vaccines as another crime against humanity.

Notably, we are regretful that many "honorable" journals, including one affiliated to the CDC, have refused to peer review this manuscript though a draft was submitted to many before the appearance of mortalities attributed to those vaccines and nothing changes when submitted to other journals after these mortalities have been discovered; no opportunity for a nonbiased peer review was granted. Ironically, nothing is comparable to this misfortunate dishonorable academic misconduct, as I suggest, except the intended one year persistent denial, by dozens of similarly "reputable" journals to fairly review our immunomodulatory protocol that provides a safe, inexpensive cure to COVID-19 and even when it was accepted after peer review, some have intervened to remove it from publication and I am so fortunate to have it recently published [79].

Moreover, We urge the CDC to consider a change to its current recommendation to advise against use of nucleic acid based vaccination for COVID-19 patients complaining of autoimmune diseases and we suggest that FDA should investigate a potential extra risk that might be associated with BNT162b2 vaccine and calling for an independent re-evaluation of the post vaccination situation in Israel and we totally agree with the EMA and FDA decisions to reevaluate the safety of ChAdOx1 nCOV-19 and Ad26. COV2-S vaccines, respectively. Furthermore, that risk benefit ratio from administering convalescent plasma to COVID-19 patients might be outbalanced due to potential early ADE and a strict system for post vaccination surveillance must be secured to report any encountered serious adverse effect especially for those who would be reinfected with SARS CoV-2 despite vaccination.

Additionally, the techniques used in development of all types of SARS CoV-2 vaccines, especially the newly emergency approved ones, should focus on innovative methods to decrease their potential autoimmunity and antibody dependent disease enhancement. Finally, in all cases we believe that more careful consideration of these potential hazards must have been thoroughly discussed and/or refuted before a mass vaccination approval was granted under the cover of a so called "emergency" use approval as the public, which has been repeatedly denied a constitutional right to know, might not accept to sacrifice a minority of unaware recipients who experienced the presented serious adverse effects and/or mortalities who were denied their legal right to know first then decide.

It should also be agreeable that no matter if SARS CoV-2 vaccines associated serious adverse effects are rare as frequently officially claimed or not so rare at all as non-peer reviewed extensive analysis might reveal, the right to know then freely decide is a must. Unfortunately, it is not yet excluded that political and/or economic gains might have shared to induce a manmade Hades, currently as in India or soon in other countries that 
might currently proclaim triumph, and we wish to remind all stakeholders that no prior agreements will, ever, secure impunity yet for the sake of millions of innocents who have been vaccinated though not in the high risk groups, I pray that the once called free world might wake up soon for the best interests of humans and humanity.

\section{Acknowledgments}

This work is dedicated to Egypt; a country that I am proud to be one of its citizens, Africa; the continent that has suffered oppression for centuries and especially to my beloved mother to whom I owe my endurance and faith. The author would also like to sincerely thank Dr. James Lyons-Weiler (The Institute for Pure and Applied Knowledge; US) who after reading a preprint has generously emailed three valuable immunology related references that have enriched this and other manuscripts and I am also very grateful to Dr. Viera Malachovska, Ing, PhD a Slovakian scientist who has generously emailed precious links that discusses the safety concerns related to the BNT162b2 vaccine in Israel. I am also very much indebted to the precious feedback that has reached me from several individuals from many countries who have generously shared their reasonable doubt, fear and hopes after reading some preprints of this manuscript and I would like to thank all free men/women who dared to fight against global corruption related to this subject despite the disgraceful defaming and fully funded campaigns.

Finally, no words would ever suffice to express my utmost gratitude and deep respect to International Journal of Pulmonary \& Respiratory Sciences and to this article managing editor Ms. Elena Ramirez, at last I found an honorable destination that healed my wounds.

\section{References}

1. Kochhar S, Salmon DA (2020) Planning for COVID-19 vaccines safety surveillance. Vaccine 38(40): 6194-6198.

2. Petousis-Harris H (2020) Assessing the Safety of COVID-19 Vaccines: A Primer. Drug Safety 43(12): 1205-1210.

3. Shakir S, Lane S, Davies M (2021) How to Investigate a Serious Adverse Event Reported During a Clinical Trial for a COVID-19 Vaccine. Drug Safety 44(1): 1-5.

4. Walach H, Klement RJ, Aukema W (2021) The Safety of COVID-19 Vaccinations-We Should Rethink the Policy. Vaccines 9(7): 693.

5. Takuva S, Takalani A, Garrett N, Ameena G, Jonny P, et al. (2021) Thromboembolic Events in the South African Ad26.COV2. S Vaccine Study. N Eng J Med 385(6): 570-571.

6. Heidi Ledford (2020) US authorization of first COVID vaccine marks new phase in safety monitoring. Nature 588(7838): 377-378.

7. Madhi SA, Baillie V, Cutland CL, Merryn V, Anthonet LK et al. (2021) Safety and efficacy of the ChAdOx1 nCoV-19 (AZD1222) Covid-19 vaccine against the B.1.351 variant in South Africa. medRxiv.

8. Bleier BS, Ramanathan M, Lane AP (2021) COVID-19 Vaccines May Not Prevent Nasal SARS-CoV-2 Infection and Asymptomatic Transmission. Otolaryngol Head Neck Surg 164(2): 305-307.
9. Kelleni M. SARS CoV-2 adenovirus and RNA based vaccines potential autoimmune complications: could we lower the chances? Authorea (Preprint) 2020.

10. Lyons-Weiler J (2020) Pathogenic priming likely contributes to serious and critical illness and mortality in COVID-19 via autoimmunity. J Transl Autoimmun 3: 100051.

11. Coffman RL, Sher A, Seder RA (2010) Vaccine adjuvants: putting innate immunity to work. Immunity 33(4): 492-503.

12. Ludwig Burger (2020) AstraZeneca pauses coronavirus vaccine trial as participant illness investigated. Reuters.

13. Matthew Herper (2020) Covid-19 vaccine trials from AstraZeneca, Johnson \& Johnson to restart. Statnews.

14. Smriti M, Heidi Ledford (2020) COVID-vaccine results are on the wayand scientists' concerns are growing. Nature 586(7827):16-17.

15. (2020) J\&J pauses COVID-19 vaccine trials due to unexplained illness in participant. Reuters.

16. Rebecca R, Benjamin M (2020) After Admitting Mistake, AstraZeneca Faces Difficult Questions About Its Vaccine. The New York Times.

17. Wagner TE, Becraft JR, Bodner K, Brian Teague, Xin Zhang, et al. (2018) Small-molecule-based regulation of RNA-delivered circuits in mammalian cells. Nature Chemical Biology 14(11): 1043-1050.

18. Wen Y-M, Mu L, Shi Y (2016) Immunoregulatory functions of immune complexes in vaccine and therapy. EMBO Mol Med 8(10): 1120-1133.

19. Zhang C, Maruggi G, Shan H, Junwei Li (2019) Advances in mRNA Vaccines for Infectious Diseases. Front Immunol 10: 594.

20. Pardi N, Hogan MJ, Porter FW, Drew Weissman (2018) mRNA vaccinesa new era in vaccinology. Nat Rev Drug Discov 17(4): 261-279.

21. Andries O, Kitada T, Bodner K, Niek NS, Ron Weiss (2015) Synthetic biology devices and circuits for RNA-based 'smart vaccines': a propositional review. Expert Rev Vaccines 14(2): 313-331.

22. Wang F, Kream RM, Stefano GB (2020) An Evidence Based Perspective on mRNA-SARS-CoV-2 Vaccine Development. Med Sci Monit 26: e924700.

23. Bloom K, Vanden Berg F, Arbuthnot P (2020) Self-amplifying RNA vaccines for infectious diseases. Gene Ther 28(3-4): 117-129.

24. (2021) Zusammenhang unwahrscheinlich: Institut prüft zehn Todesfälle nach Impfung [German]. NTV

25. Guo L, Rondina MT (2019) The Era of Thromboinflammation: Platelets Are Dynamic Sensors and Effector Cells During Infectious Diseases. Front Immunol 10: 2204.

26. Hoffman M, Monroe DM, Roubey RA (2000) Links between the immune and coagulation systems: how do "antiphospholipid antibodies" cause thrombosis? Immunol Res 22(2-3): 191-197.

27. Engelmann B, Massberg S (2013) Thrombosis as an intravascular effector of innate immunity. Nat Rev Immunol 13(1): 34-45.

28. Tamaki H, Khasnis A (2015) Venous thromboembolism in systemic autoimmune diseases: A narrative review with emphasis on primary systemic vasculitides. Vasc Med 20(4): 369-376.

29. Aggarwal S, Sharma N (2013) Cerebral venous sinus thrombosis with autoimmune thyroiditis. Indian J Endocrinol Metab 17(Suppl 1): S176-S177.

30. Carhuapoma JR, Mitsias P, Levine SR (1997) Cerebral Venous Thrombosis and Anticardiolipin Antibodies. Stroke 28(12): 23632369. 
31. Greinacher A, Thiele T, Warkentin TE, Karin W, Paul AK, et al. (2021) Thrombotic Thrombocytopenia after ChAdOx1 nCov-19 Vaccination. N Engl J Med 384(22): 2092-2101.

32. See I, Su JR, Lale A, Emily JW, Alice YG, et al. (2021) US Case Reports of Cerebral Venous Sinus Thrombosis With Thrombocytopenia After Ad26.COV2.S Vaccination, March 2 to April 21, 2021. JAMA 325(24): 2448-2456.

33. Maxime T, Masud H, John RG, Sierra L, Paul JH (2021) Cerebral venous thrombosis: a retrospective cohort study of 513,284 confirmed COVID-19 cases and a comparison with 489,871 people receiving a COVID-19 mRNA vaccine.

34. Bracamonte-BW, Cihakova D (2017) Cardiac Autoimmunity: Myocarditis. Adv Exp Med Biol 1003: 187-221.

35. Khalil A, Naneishvili T, Prasad N, James Glancy (2021) SARS-CoV-2 myocarditis: what physicians need to know. BMJ Evid Based Med bmjebm-2020-111527.

36. Esfandiarei M, Mc Manus BM (2008) Molecular Biology and Pathogenesis of Viral Myocarditis. Annu Rev Pathol 3: 127-155.

37. Savvatis K, Muller I, Frohlich M, Kathleen P, Christin Z, et al. (2014) Interleukin- 6 receptor inhibition modulates the immune reaction and restores titin phosphorylation in experimental myocarditis. Basic Res Cardiol 109(6): 449.

38. Kelleni MT (2021) NSAIDs/Nitazoxanide/Azithromycin Repurposed for COVID-19: Potential Mitigation of the Cytokine Storm Interleukin-6 Amplifier via Immunomodulatory Effects. Expert Rev Anti Infect Ther Pp: 1-5.

39. Saleh E, Moody MA, Walter EB (2016) Effect of antipyretic analgesics on immune responses to vaccination. Hum vaccin Immunother 12(9): 2391-2402.

40. Karaderi T, Bareke H, Kunter I, Adil S, Ilgin C, et al. (2020) Host Genetics at the Intersection of Autoimmunity and COVID-19: A Potential Key for Heterogeneous COVID-19 Severity. Front Immunol 11: 586111.

41. Kira Smith (2021) BNT162b2 Vaccine: Possible Codons Misreading Errors in Protein Synthesis and Alternative Splicing's Anomalies. Journal of Antivirals \& Antiretrovirals 13(2).

42. Karthik K, Senthilkumar TMA, Udhayavel S, Gopal DR (2020) Role of antibody-dependent enhancement (ADE) in the virulence of SARS CoV-2 and its mitigation strategies for the development of vaccines and immunotherapies to counter COVID-19. Hum Vaccin Immunother 16(12): 3055-3060.

43. Yip MS, Leung NHL, Cheung CY, Ping HL, Horace HYL, et al. (2014) Antibody-dependent infection of human macrophages by severe acute respiratory syndrome coronavirus. Virol J 11: 82.

44. Tetro JA (2020) Is COVID-19 receiving ADE from other coronaviruses? Microbes Infect. 22(2): 72-73.

45. Adrielle dos SL, Filho PGdG, Silva AMF, Joao VGS, Douglas SS, et al (2021) Recurrent COVID-19 including evidence of reinfection and enhanced severity in thirty Brazilian healthcare workers. J Infect 82(3): 399-406

46. Hansen T, Titze U, Kulamadayil-HNSA, Sabine Glom, Johannes JT, et al. (2021) First case of postmortem study in a patient vaccinated against SARS-CoV-2. Int J Infect Dis107: 172-175.

47. Lee WS, Wheatley AK, Kent SJ, Brandon JDK (2020) Antibodydependent enhancement and SARS-CoV-2 vaccines and therapies. Nat Microbiol 5(10): 1185-1191.

48. Liu L, Wei Q, Lin Q, Jun F, Haibo W, et al. (2019) Anti-spike IgG causes severe acute lung injury by skewing macrophage responses during acute SARS-CoV infection. JCI Insight 4(4): e123158.

49. Tseng CT, Sbrana E, Iwata-Yoshikawa N, Patrick CN, Tania G, et al. (2012) Immunization with SARS coronavirus vaccines leads to pulmonary immunopathology on challenge with the SARS virus. PLoS One 7(4): e35421.

50. Yip MS, Cheung CY, Li PH, Roberto B, JSM Peiris, et al. (2011) Investigation of Antibody-Dependent Enhancement (ADE) of SARS coronavirus infection and its role in pathogenesis of SARS. BMC Proceedings 5(1): P80.

51. Farshi E (2020) Cytokine Storm Response to COVID-19 Vaccinations Journal of Cytokine Biology 5: 1000125.

52. Fu Y, Cheng Y, Wu Y (2020) Understanding SARS-CoV-2-Mediated Inflammatory Responses: From Mechanisms to Potential Therapeutic Tools. Virol Sin 35(3): 266-271.

53. Quinlan BD, Mou H, Zhang L, Yan G, Wenhui H, et al. (2020) The SARS$\mathrm{CoV}-2$ receptor-binding domain elicits a potent neutralizing response without antibody-dependent enhancement. bioRxiv.

54. Iwasaki A, Yang Y (2020) The potential danger of suboptimal antibody responses in COVID-19. Nat Rev Immunol 20(6): 339-341.

55. Yu J, Tostanoski LH, Peter L, Noe BM, Katherine MM, et al. (2020) DNA vaccine protection against SARS-CoV-2 in rhesus macaques. Science 369(6505): 806-811.

56. Arvin AM, Fink K, Schmid MA, Andrea C, Roberto S, et al. (2020) A perspective on potential antibody-dependent enhancement of SARSCoV-2. Nature 584(7821): 353-363

57. Pathak EB (2020) Convalescent plasma is ineffective for covid-19. BM] 371: $\mathrm{m} 4072$.

58. Mejias A, Rodriguez FR, Oliva S, Mark EP, Octavio R (2020) The journey to a respiratory syncytial virus vaccine. Ann Allergy Asthma Immunol 125(1): 36-46.

59. Fulginiti VA, Eller JJ, Downie AW, C H Kempe (1967) Altered Reactivity to Measles Virus: Atypical Measles in Children Previously Immunized with Inactivated Measles Virus Vaccines. JAMA 202(12): 1075-1080.

60. Amit S, Sharon AB, Biber A, Grinberg A, Leshem E, et al. (2021) Postvaccination COVID-19 among healthcare workers, Israel. Emerg Infect Dis 27(4): 1220-1222.

61. Williams TC, Burgers WA (2021) SARS-CoV-2 evolution and vaccines: cause for concern? The Lancet Respiratory Medicine 9(4): 333-335.

62. Kelleni M (2021) SARS CoV-2 Vaccines, Remdesivir and Favipiravir Might Have Led to SARS CoV-2 B.1.617 Variants: India First but We Can Intervene.

63. Angum F, Khan T, Kaler J, Lena S, Azhar H (2020) The Prevalence of Autoimmune Disorders in Women: A Narrative Review. Cureus 12(5): e8094.

64. Hussain MS, Tripathi V (2018) Smoking under hypoxic conditions: a potent environmental risk factor for inflammatory and autoimmune diseases. Mil Med Res 5(1): 11

65. Adrish M, Chilimuri S, Mantri N, Haozhe S, Maleeha Z, et al. (2020) Association of smoking status with outcomes in hospitalised patients with COVID-19. BMJ Open Respir Res 7(1): e000716.

66. Jimenez RCA, Lopez PD, Alonso AA, Rafael AB, Segismundo SR, et al. (2021) COVID-19 and Smoking: A Systematic Review and MetaAnalysis of the Evidence. Arch Bronconeumol 57 (Suppl 1): 21-34.

67. Patanavanich R, Glantz SA (2020) Smoking is associated with worse outcomes of COVID-19 particularly among younger adults: A systematic review and meta-analysis. medRxiv. 
68. Usman MS, Siddiqi TJ, Khan MS, Urvish KP, Izza Shahid, et al. (2020) Is there a smoker's paradox in COVID-19? BMJ Evidence-Based Medicine.

69. Carmona BA, Jimenez FP, Sanchez AA, Felipe AM, Eduardo C (2020) Does active smoking worsen Covid-19? Eur J Intern Med 77: 129-131.

70. Versini M, Jeandel PY, Rosenthal E, Yehuda S (2014) Obesity in autoimmune diseases: not a passive bystander. Autoimmun Rev 13(9): 981-1000.

71. Rogers MAM, Wei MY, Kim C, Joyce ML (2020) Sex Differences in Autoimmune Multimorbidity in Type 1 Diabetes Mellitus and the Risk of Cardiovascular and Renal Disease: A Longitudinal Study in the United States, 2001-2017. J Womens Health (Larchmt) 29(4): 511-519.

72. Figliozzi S, Masci PG, Ahmadi N, Lara T, Evangelia K, et al. (2020) Predictors of adverse prognosis in COVID-19: A systematic review and meta-analysis. Eur J Clin Invest 50(10): e13362.

73. Williamson E, Walker AJ, Bhaskaran KJ, Seb Bacon, Chris Bates, et al. (2020) OpenSAFELY: factors associated with COVID-19-related hospital death in the linked electronic health records of 17 million adult NHS patients. medRxiv.
74. Yang S, Liu T, Liang G (2020) The benefits of smoking cessation on survival in cancer patients by integrative analysis of multi-omics data. Mol Oncol 14(9): 2069-2080.

75. (2020) Coronavirus Disease 2019 (COVID-19): People with Certain Medical Conditions. CDC.

76. Grant A, Hunter PR (2021) Immunisation, asymptomatic infection, herd immunity and the new variants of COVID 19. medRxiv.

77. Shimabukuro TT, Kim SY, Myers TR, Pedro LM, Titilope O, et al. (2021) Preliminary Findings of mRNA Covid-19 Vaccine Safety in Pregnant Persons. N Eng J Med 384(24): 2273-2282.

78. Kelleni MT (2021) Tocilizumab, Remdesivir, Favipiravir, and Dexamethasone Repurposed for COVID-19: a Comprehensive Clinical and Pharmacovigilant Reassessment. SN Compr Clin Med Pp: 1-5.

79. Kelleni MT (2021) NSAIDs/Nitazoxanide/Azithromycin Immunomodulatory Protocol Used in Adult, Geriatric, Pediatric Pregnant, and Immunocompromised COVID-19 Patients: A Real-World Experience. Canadian Journal of Medicine 3(3): 121-143.

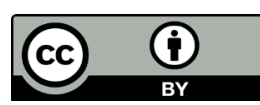

This work is licensed under Creative Commons Attribution 4.0 License

DOI: 10.19080/IJOPRS.2021.05.555658
Your next submission with Juniper Publishers will reach you the below assets

- Quality Editorial service

- Swift Peer Review

- Reprints availability

- E-prints Service

- Manuscript Podcast for convenient understanding

- Global attainment for your research

- Manuscript accessibility in different formats ( Pdf, E-pub, Full Text, Audio)

- Unceasing customer service

Track the below URL for one-step submission https://juniperpublishers.com/online-submission.php 